\title{
Direct observation of the tube model in F-actin solutions: Tube dimensions and curvatures
}

\author{
M. Romanowska ${ }^{1,2}$, H. Hinsch ${ }^{3}$, N. Kirchgessner ${ }^{1}$, M. Giesen ${ }^{1}$, M. Degawa $^{4}$, B. Hoffmann ${ }^{1}$, E. Frey ${ }^{3}$ \\ nd R. MERKEL ${ }^{1}$ \\ Institute of Bio- and Nanosystems 4: Biomechanics, Research Centre Jülich, 52425 Jülich, Germany \\ Marian Smoluchowski Institut of Physics, Jagiellonian University Kraków, Reymonta 4, 30-059 Kraków, Poland \\ Arnold Sommerfeld Center for Theoretical Physics and Center for Nanoscience, Ludwig-Maximilians-Universität \\ München, 80333 Munich, Germany \\ Laboratory of Molecular Dynamics, Brain Science Institute, RIKEN, Wako, Saitama 351-0198, Japan
}

PACS 61.25.H - Macromolecular and polymer solutions

PACS 82.35.Pq - Biopolymers

PACS 87.16.Ln - Cytoskeleton

\begin{abstract}
Mutual uncrossability of polymers generates topological constraints on their conformations and dynamics, which are generally described using the tube model. We imaged confinement tubes for individual polymers within a F-actin solution by sampling over many successive micrographs of fluorescently labeled probe filaments. The resulting average tube width shows the predicted scaling behavior. Unexpectedly, we found an exponential distribution of tube curvatures which is attributed to transient entropic trapping in network void spaces.
\end{abstract}

Introduction. - One of the central questions of polymer physics is how the properties of polymeric materials, uch as fluids or gels, derive from the characteristics of he individual macromolecules forming the material. In olymer solutions, entanglements between neighboring filaments impose topological constraints on the Brownian motion of individual polymers. These constraints, however, are notoriously difficult to incorporate into a theoretical description of the system. Arguably the most successful phenomenological approach to this problem is the tube model introduced by Edwards [1]. It reduces the complex many-filament problem to a tractable single-filament problem, where the topological constraints imposed by the surrounding polymers are condensed to the concept of an impenetrable tube. This assumption is at the heart of most theories of macromolecular behavior such as the reptation model [2]. For solutions of linear polymers that are not cross-linked the confinement tube is a dynamic entity that is defined on intermediate time scales ranging from the moment of first contact between neighboring polymers to the characteristic time for tube remodelling. However, this time window can be of substantial size [2].

Up to now the tube model has remained mostly a conceptual device for calculations with few direct experimen- tal observations $[3,4]$. Due to this sparseness of data most theories on collective properties of semi-dilute or dense polymer solutions rely on the assumption that filaments are enclosed in confinement tubes with fixed widths throughout the sample. Moreover, the ensemble of confinement tubes is usually assumed to qualitatively show the same conformation statistics as an individual polymer in free solution. In the present study, we challenged these assumptions by directly imaging confinement tubes of fluorescently labeled actin filaments embedded in semi-dilute solutions of unlabeled filaments.

Actin filaments (F-actin) form by self-aggregation of the protein actin and exhibit lengths of some tens of microns. In solution, these filaments undergo thermal shape fluctuations but, due to their bending stiffness, maintain an average direction and are therefore referred to as semiflexible; their persistence length ranges from $15-20 \mu \mathrm{m}$ for phalloidin stabilized filaments [5-7] to approximately $10 \mu \mathrm{m}$ for unstabilized filaments [8]. At present, the properties of isolated filaments are reasonably well understood in terms of the wormlike chain model [9] but the relation between microstructure and viscoelastic properties of entangled solutions is still a challenging problem [10,11].

For semiflexible polymers, a typical tube is pictured as a 
thin and relatively straight cylindrical pore. A thermally fluctuating filament confined within such a tube will encounter collisions with the tube wall, whose average distance is given by Odijk's deflection length [12]. Expanding this concept Semenov derived a scaling law for the dependence of tube width, $w$, on monomer concentration, $c$ [13]. Basically, he exploited the fact that fluctuating filaments explore non-overlapping regions of space and predicted

$$
w \propto c^{-3 / 5} .
$$

In contrast, the mean distance between neighboring filaments, $\xi$, i.e. the mesh size, scales for purely geometrical reasons like $c^{-1 / 2}$. Thus mesh size and confinement tube width are genuinely different length scales. Confinement is usually described by a harmonic tube potential which has been confirmed by experiments [14] and computer simulations [15].

In this Letter, we present a quantitative study of confinement tubes in entangled F-actin networks. We fluorescently labeled a trace amount of actin filaments with rhodamine-phalloidin, embedded those in a solution of unlabeled filaments and observed individual filaments by confocal laser scanning microscopy. A typical micrograph is shown in Fig. 17a. Upon averaging time series of such images, we obtained an intensity distribution representing the probability density of a filament within its tube (Fig. 10). This approach allowed us to directly characterize the size and conformation of individual confinement tubes in real space at optical resolution.

Experimental. - Actin was prepared as described in the literature [16]. Monomeric actin was kept in G-buffer (2.0 mM Tris- $\mathrm{HCl}, \mathrm{pH} 8.0,0.2 \mathrm{mM} \mathrm{CaCl}_{2}, 0.2 \mathrm{mM}$ ATP, $0.2 \mathrm{mM}$ DTT) at $4^{\circ} \mathrm{C}$ and used for experiments within $72 \mathrm{~h}$. Polymerization was initiated by adding $1 / 10$ of the sample volume of $10 \times$ F-buffer (20 mM Tris-HCl, pH 7.5, $2 \mathrm{mM} \mathrm{CaCl}_{2}$, $5 \mathrm{mM}$ ATP, $2 \mathrm{mM}$ DTT, $20 \mathrm{mM} \mathrm{MgCl}_{2}, 1 \mathrm{M}$ $\mathrm{KCl}$ ). The samples were then incubated for $2 \mathrm{~h}$ at $37^{\circ} \mathrm{C}$. A part of the F-actin was labeled with rhodamine-phalloidin (TRITC-phalloidin) at a molar ratio of 1:1. Labeled and unlabeled F-actin solutions of the same concentration were then gently mixed at 1:1000 ratio for $15 \mathrm{~min}$ in a slow (few $\mathrm{rpm}$ ) rotary shaker. The resulting solution was gently pipetted onto a micro slide and overlayed with a cover slide which was held at a distance of $100 \mu \mathrm{m}$ by a spacer of adhesive tape. Experiments were performed after an equilibration period of $15 \mathrm{~min}$.

Filaments were observed using an inverse confocal microscope (LSM510 equipped with a water immersion objective, C-Apochromat 40/1.2W corr, Carl Zeiss, Jena, Germany). Excitation wavelength was $543 \mathrm{~nm}$. Only filaments residing in a plane perpendicular to the optical axis at a distance of $40-60 \mu \mathrm{m}$ from the cover slide were considered. The thickness of the optical slice was typically set to $2.8 \mu \mathrm{m}$. A field of view containing one labeled filament was scanned within 0.1-0.3 s. Images were taken at $1 \mathrm{~s}$ intervals within a time period of $2-3$ minutes. On
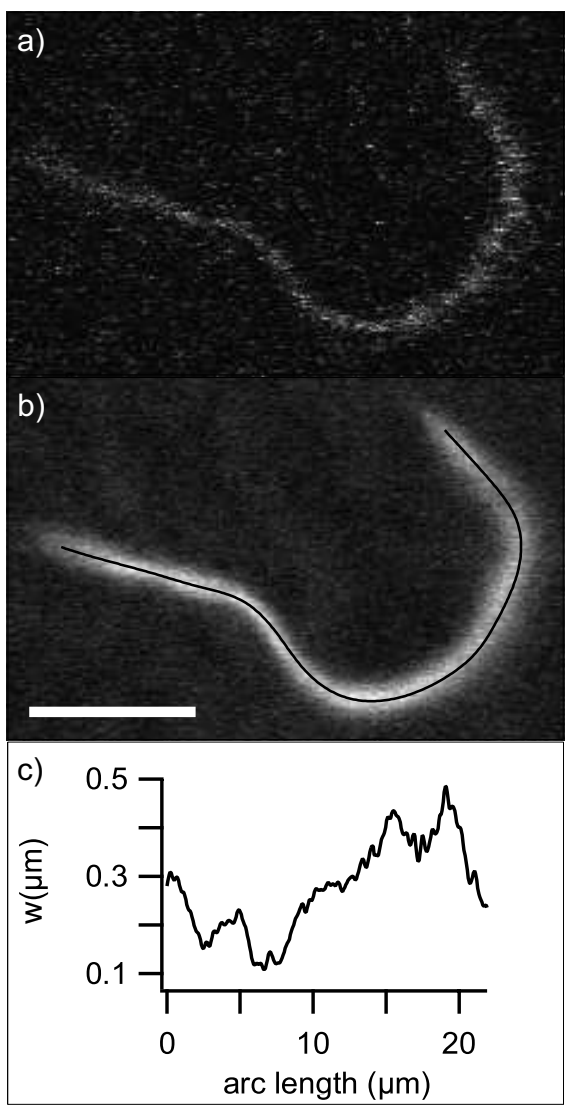

Fig. 1: (a) Micrograph of a fluorescently labeled F-actin within a solution of concentration $c=14.5 \mu \mathrm{M}$. (b) Average of 100 successive images taken within $126 \mathrm{~s}$. Overlayed is the center line of the averaged contour (black line). Scale bar $5 \mu \mathrm{m}$. (c) The width of the averaged intensity distribution corrected for optical resolution effects. Arc length runs from left to right in the micrographs.

this time scale we observed only transverse filament fluctuations. Time-lapse sequences of about 25 filaments were collected for each concentration.

As contour characterizing a confinement tube we used the center line of the intensity distribution in the time averaged micrographs, cf. Fig. 1b. We determined this contour by the following algorithm: The image was binarized with a global threshold. The resulting areas were skeletonized to obtain a first estimate of the contour at pixel accuracy. Then the end points of this line were kept fixed and an active contour algorithm based on energyminimization was applied on the time averaged micrographs as described in Ref. [17]. This resulted in a smooth line representing the contour of the confinement tube; see Fig. 10. All image processing routines were developed using MatLab (Release 14, The Mathworks, Natick, MA). The width of the confinement tube, $\sigma_{\exp }$, was determined as the standard deviation of a Gaussian fit to the intensity distribution of the averaged image along lines perpendicular to the contour. A result from this procedure is shown in Fig. 1. 


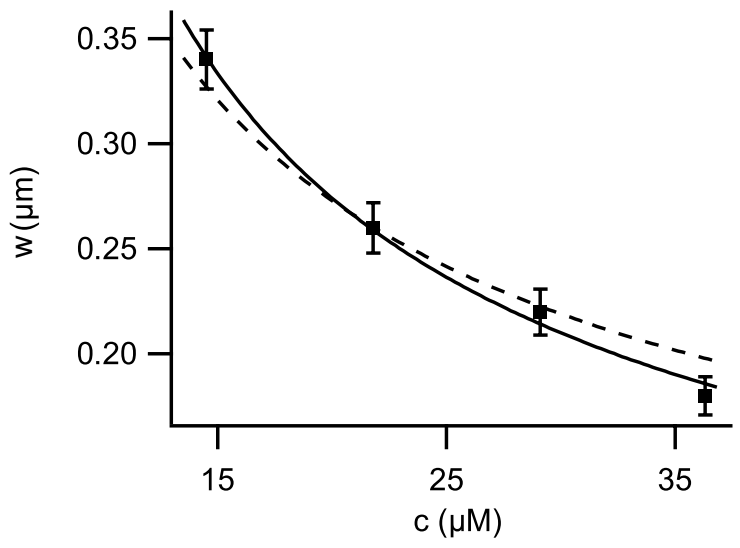

Fig. 2: Dependence of the average tube widths, $w$, on actin concentration, $c$. Symbols: measured points, error bars: standard deviations of the mean tube widths of the filaments, full line: best fit to a power law (resulting exponent -0.61), dashed line: best fit with exponent $-1 / 2$.

Experimental Results. - Our first objective was to analyze the functional dependence of the average tube width $w$ on the actin monomer concentration $c$. The data for the width $\sigma_{\text {exp }}$ were corrected for the optical resolution $\sigma_{\mathrm{LSF}}$ to give an undistorted tube width $w=$ $\left(\sigma_{\exp }^{2}-\sigma_{\mathrm{LSF}}^{2}\right)^{1 / 2}$. We also took into account that a socalled critical concentration $(1.9 \mu \mathrm{M})$ of actin monomers forms a pool of unpolymerized molecules in equilibrium with the actin filaments, i.e. in Eq. 1 the concentration of actin within filaments was taken to be $1.9 \mu \mathrm{M}$ less than the total actin concentration. Average widths of the confinement tubes were $0.34,0.26,0.22$, and $0.18 \mu \mathrm{m}$ at total actin concentrations of $14.5,21.8,29.1$, and $36.3 \mu \mathrm{M}$, respectively. These data conform quite well to a power law curve with a best fit for an exponent of -0.61, cf. Fig. 2, An exponent $-1 / 2$, as expected for the mesh size, is clearly not compatible with our data. To our knowledge, this result is the first experimental confirmation of the predicted Semenov scaling exponent of $-3 / 5$, Eq. 1, showing that indeed tube width and mesh size are distinct length scales [13]. Moreover, even the absolute values of the tube width as predicted by recent theoretical studies $[15,18]$ are of comparable but lower magnitude. This is consistent since these treatments do not include collective fluctuations of the entangled solution and therefore give lower bounds.

So far our results nicely confirmed previous theoretical predictions on the average tube width. However, contrary to common belief, fluctuations of the tube widths were pronounced with standard deviations of $\approx 35 \%$; cf. Fig. 固. The length scale over which tube width fluctuations occurred was studied via the autocorrelation function of

\footnotetext{
${ }^{1}$ The optical resolution was calculated by convoluting the point spread function measured on $100 \mathrm{~nm}$ fluorescent beads with a line. The resulting line spread function (LSF) was well approximated by a Gaussian of width $\sigma_{\mathrm{LSF}}$, which on average was $0.18 \mu \mathrm{m}$
}

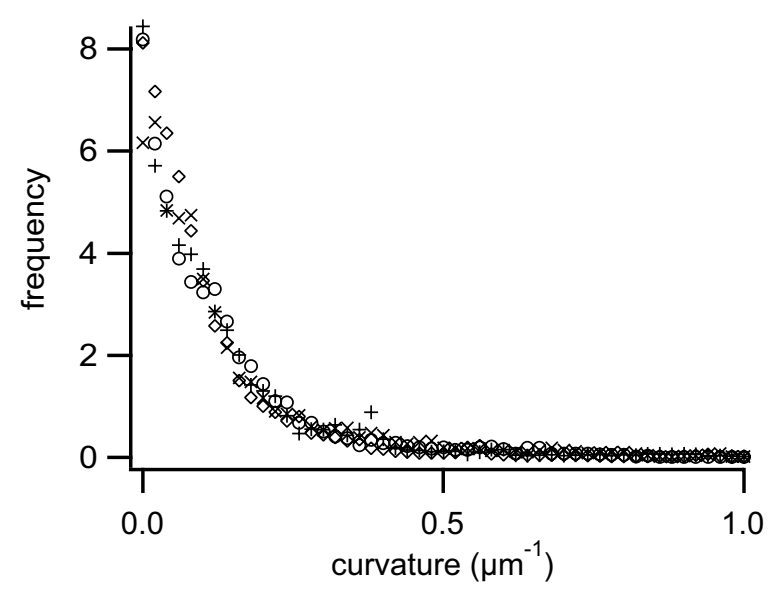

Fig. 3: Distribution functions of the curvatures of the confinement tubes. $+36.3 \mu \mathrm{M}, \times 29.1 \mu \mathrm{M}, \circ 21.8 \mu \mathrm{M}$, and $\diamond 14.5 \mu \mathrm{M}$.

$\sigma_{\text {exp }}$, defined as $A C F(j)=\frac{\sum_{i}^{N}\left(\sigma_{i}-<\sigma>\right)\left(\sigma_{i+j}-<\sigma>\right)}{\sum_{i}^{N}\left(\sigma_{i}-<\sigma>\right)\left(\sigma_{i}-<\sigma>\right)}$. In this equation the subscript "exp" was dropped for the sake of brevity. The initial decay parameters of the autocorrelation functions of $\sigma_{\exp }$ were $0.33,0.49,0.60,0.68 \mu \mathrm{m}^{-1}$ at concentrations of $14.5,21.8,29.1$, and $36.3 \mu \mathrm{M}$, respectively. Thus tube width fluctuations occurred on length scales of few microns. The faster decay of correlations in the tube width at higher concentrations is a consequence of the higher obstacle density.

To go beyond a characterization of a tube in terms of its typical size we investigated tube conformations. Specifically, we asked for the probability distribution function of tube curvatures. In the absence of excluded volume it seems obvious that the probability distribution of polymer conformations in solution is identical to that of free filaments. Sometimes, it is argued that even the (coarsegrained) conformations of the tube contour should follow such a free distribution [19]. Since the effective free energy of the wormlike chain model is quadratic in the local curvature this would imply a Gaussian distribution for the curvatures of confinement tubes. To our great surprise we found a curvature distribution with a pronounced exponential tail; see Figs. 3 and 4 top.

The initial decay length of the curvature distribution shows little to no dependence on concentration; the decay lengths of the distributions were 8.0, 8.0, 8.2, and $9.9 \mu \mathrm{m}$ at the concentrations of $36.3,29.1,21.8$, and $14.5 \mu \mathrm{M}$, respectively. This is to be expected since small curvatures are dominated by bending stiffness.

An exponential distribution decays much slower towards large values than a Gaussian. This is reflected in our frequent observation of highly bent filaments. For isolated filaments, similarly bent contours are extremely rare and were never observed [5-7]. What is causing such a qualitative change in chain conformations? At this point several reasons for the occurrence of highly bent shapes, besides topological interactions, were conceivable. We could 
rule out that filaments assumed such conformations during sample preparation and remained kinetically trapped by additional experiments where the time delay between successive micrographs of actin filaments was set in the range from 10 to $30 \mathrm{sec}$. With these longer time delays we were able to track individual filaments for up to one hour. On these time scales we observed several initially straight filaments going into highly bent shapes. This observation indicates that the highly bent shapes causing the exponential decay of the distribution of curvatures of the confinement tubes are an intrinsic property of the system and not just kinetically trapped remainders of the preparation procedure.

Simulation. - We resorted to Monte Carlo simulations to elucidate the underlying causes for the observed exponential distribution of tube curvatures. We considered an initially equilibrated test polymer in a twodimensional observation plane surrounded by randomly placed point-like obstacles representing the intersection points of neighboring polymers with that very plane. The tube contour was obtained by observing the test polymer's fluctuations in the presence of harmonically fluctuating point obstacles. Since these obstacles represent themselves polymers with identical properties, we tuned the fluctuation amplitudes of the obstacles to equal those of the test polymer to obtain a fully self-consistent model. Sampling over filaments in different network configurations and averaging over all statistically allowed conformations in a given tube resulted in a curvature distribution that also featured a pronounced exponential tail. As this tail shows no significant dependence on concentration in the experimental parameter range we compare the simulation results to the combined experimental data for all concentrations and find good agreement; see Fig. 目 top.

We therefore concluded that the observed exponential distribution of tube curvatures is indeed characteristic for semi-dilute solutions of semiflexible polymers. Compared to free filaments, both smaller and larger curvatures are more frequent. An increased frequency of small curvatures is due to the coarse-graining procedure involved in determining the tube contour: averaging over all topologically allowed polymer conformations within the tube (simulations) or performing a time average (experiment) results in a smoother contour and shifts weight of the probability distribution towards small curvatures. The origin for the relatively large number of highly bent filaments and concomitantly the exponential tail of the curvature distribution is far less obvious. To avoid complications from coarse-graining we analyzed the distribution function of the confined filament itself. Even though not easily accessible to experiments like the tube contour, it allows a direct comparison to free filaments. Again, a fully self-consistent simulation of the polymer configuration resulted in an exponential tail in the curvature dis-

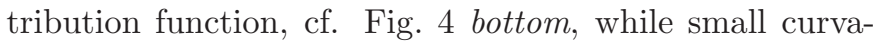
tures remained unaffected. Monitoring polymer segments

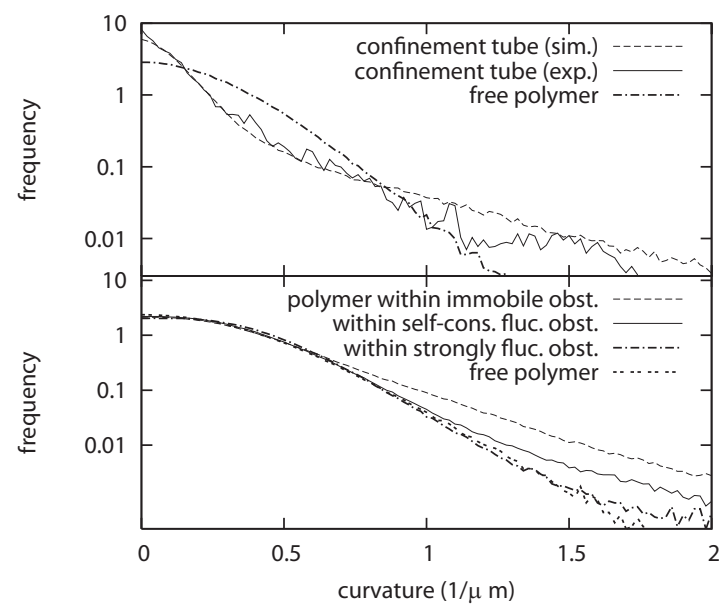

Fig. 4: Top: Curvature distribution of confinement tubes of probe filaments in a dynamic network (experimental data and simulations) and distribution function of a free polymer (simulations). Bottom: Simulated curvature distributions of free filaments, filaments within a self-consistently fluctuating network, within a network of fixed and a network of strongly fluctuating obstacles.

with unusual high curvatures during the simulation enabled us to attribute this effect to network environments where a favorably bent test polymer can protrude into a large void space thereby realizing a higher entropy 2 . Upon immobilizing the obstacles the effect is enhanced, while for

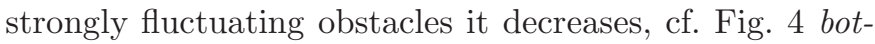
tom. This is due to a smaller effective void volume and is expected to recover the free polymer case when strong obstacle delocalization renders the network comparable to a gas. The phenomenon bears some similarity with "entropic trapping" [20,21] observed for flexible polymers in random environments.

Discussion. - Clearly, in the absence of excluded volume, time averaging of a single test polymer over infinite time as well as averaging over an ensemble of polymers at a given time both have to reproduce the Gaussian distribution of free filaments. The fact that our results do not reflect this thermodynamic equilibrium might be disturbing at first glance, but on closer inspection turns out to be a consequence of the tube concept and the time scales involved.

It is important to realize that for a solution of not cross-linked polymers the tube model itself is a concept to describe polymer dynamics and not equilibrium polymer conformations. In polymer solutions the confinement tube of a given polymer is defined as the space explored by the polymer's fluctuations on intermediate time scales ranging from the moment when the polymer first experiences topological restrictions by nearest neighbors to the time scale of tube remodelling. Both points in time can be estimated from experiment. In dynamic light scattering a

\footnotetext{
${ }^{2} \mathrm{H}$. Hinsch and E. Frey, to be published
} 
Direct observation of the tube model in F-actin solutions

cross-over from single filament to restricted dynamics is seen at about $10 \mathrm{msec}[22]$. The time scale of tube remodelling can be estimated from experiments on actin reptation by the Sackmann group [23, 24]. From their data we deduced reptation times of $15 \mathrm{~min}$ for a $1 \mu \mathrm{m}$ filament, 9 days for a $10 \mu \mathrm{m}$ filament and 3 years for a $50 \mu \mathrm{m}$ filament. As reliable curvature determination requires a polymer length of at least $8 \mu \mathrm{m}$, experimental observation of terminal relaxation by reptation is entirely impossible. In essence the same holds for a molecular dynamics simulation (even with coarse grained models) of the process where simulation times by far exceed the possibilities of even the most recent supercomputers due to the enormous number of different topologies. Interestingly, for actin filaments of some micrometer length the often neglected treadmilling motion occurring at a rate of approximately $2 \mu \mathrm{m}$ per hour $[25,26]$ should dominate terminal relaxation. Yet, even a relaxation time scale of 5 hours for a $10 \mu \mathrm{m}$ filament is far beyond the time window of light microscopic observations on such delicate samples. From these literature data we estimate the time window during which a confinement tube is well defined to range from $10 \mathrm{msec}$ to 5 hours for not stabilized actin filaments of $10 \mu \mathrm{m}$ length. The latter value increases to 9 days for stabilized actin networks where the treadmilling motion of actin is abolished by phalloidin. These values demonstrate that actin filaments in solution are trapped in their localization tubes during almost the entire experimentally accessible time window. Consequently, all observations on this time scale are crucially influenced by the tube's properties.

This also holds for the average performed in both experiments and simulations where we start from an ensemble of initial configurations of test polymers embedded in the network, and sample their configurations over a given finite time window. Hereby we avoid the infeasible sampling of the complete phase space with one slowly reptating test polymer, but for each initial condition we sample only over the part of phase space that is represented by the associated localization tube. The ensemble has to be sufficiently large to incorporate all relevant areas of the phase space. Each member of this ensemble may now be characterized in terms of its "topology" given by the topological constraints imposed by its neighbors. These topologies represent a partitioning of phase space. If the dynamics over the finite time window would conserve the topology, sampling again would necessarily have to give a Gaussian distribution. Actually, however, the polymer dynamics inside their localization tubes as well as the Markovian dynamics employed in the simulations are metrically transitive, i.e. the partitioning is not maintained under its dynamics. In particular, local network configurations where large void spaces are explored as explained above by means of reptation or "breathing" result in axial motion of the polymer's ends and thus allow for a modification of the topological partitioning. Hence, entropic trapping and the concomitantly highly bent configurations of filaments and tubes are intimately connected to transient non-equilibrium distribution functions that are observed on time scales well below large scale reptation sets in.

In summary, we studied the conformations of confinement tubes in entangled solutions of semiflexible polymers by direct visualization. Our results show that confinement tubes are very real objects for these systems. The dependence of the average tube width on actin concentration conforms to Semenov's scaling prediction [13]. In experiment and simulation we identified coinciding exponential tails in the curvature distribution of the confinement tubes. This deviation is due to transient entropic trapping and is observed on time scales below large scale reptation. These findings quantify and extend the tube concept and demonstrate that solutions of entangled semiflexible polymers are not accurately described by standard concepts of equilibrium thermodynamics on experimentally relevant time scales.

$$
* * *
$$

We thank I. Lauter, IBN-4, for insightful discussions of the manuscript and control measurements. E.F. and H.H. acknowledge support from the DFG through SFB 486, from the German Excellence Initiative via the NIM program and from the Elite Network of Bavaria through the NBT program. M.D. is grateful for support from the Alexander von Humboldt Foundation.

\section{REFERENCES}

[1] Edwards S. F., Proc. Phys. Soc., 92 (1967) 9.

[2] De Gennes P. G., Scaling Concepts in Polymer Physics (Cornell University Press, Ithaca and London) 1979.

[3] Kas J., Strey H. and Sackmann E., Nature, 368 (1994) 226.

[4] Schleger P., Farago B., Lartigue C., Kollmar A. and Richter D., Phys. Rev. Lett., 81 (1998) 124.

[5] Ott A., Magnasco M., Simon A. and Libchaber A., Physical Review E , 48 (1993) R1642.

[6] Gittes F., Mickey B., Nettleton J. and Howard J., Journal of Cell Biology, 120 (1993) 923.

[7] Le Goff L., Halatschek O., Frey E. and Amblard F., Physical Review Letters, 89 (2002) .

[8] Isambert H., Venier P., Maggs A. C., Fattoum A., Kassab R., Pantaloni D. and Carlier M.-F., The Journal of Biological Chemistry, 270 (1995) 11437.

[9] Wilhelm J. and Frey E., Physical Review Letters , 77 (1996) .

[10] Shin J. H., Gardel M. L., Mahadevan L., MatsuDaira P. and Weitz D. A., PNAS U.S.A., 101 (2004) 9636.

[11] Liu J., Gardel M. L., Kroy K., Frey E., Hoffman B. D., Crocker J. C., Bausch A. R. and Weitz D. A., Phys. Rev. Lett., 96 (2006) 118104.

[12] ODisk T., Macromolecules, 16 (1983) 1340.

[13] Semenov A. N., J. Chem. Soc., Faraday Trans. 2,82 (1986) 317. 
[14] Dichtl M. A. and Sackmann E., New Journal of Physics , (1999) 18.1.

[15] Hinsch H., Wilhelm J. and Frey E., Eur. Phys. J. E, 24 (2007) 35.

[16] Maclean-Fletcher S. and Pollard T. D., Biochemical and Biophysical Research Communications , 96 (1980) 18.

[17] Amini A. A., Weymouth T. E. and Jain R. C., IEEE Transactions on Pattern Analysis and Machine Intelligence, 12 (1990) 855.

[18] Morse D. C., Physical Review E , 63 (2001) 031502.

[19] Doi M., J. Polym. Sci. Polm. Symp. , 73 (1985) 93.

[20] Cates M. E. and Ball R. C., J. Phys. France, 49 (1988) 2009.

[21] Baumgartner A. and Muthukumar M., J. Chem. Phys. , 87 (1987) 3082.

[22] Semmrich C., Storz T., Glaser J., Merkel R., Bausch A. R. and Kroy K., Proc. Natl. Acad. Sci. USA , 104 (2007) 20199.

[23] Dichtl M. A. and Sackmann E., Proc. Natl. Acad. Sci. USA, 99 (2002) 6533.

[24] Keller M., Tharmann R., Dichtl M. A., Bausch A. R. and Sackmann E., Phil. Trans. Roy. Soc. London A , 361 (2003) 699 .

[25] Wegner A., Journal of Molecular Biology , 161 (1982) .

[26] Selve N. and Wegner A., Journal of Molecular Biology 187 (1986) 627. 